\title{
r-Sm14 - pRSETA Efficacy in Experimental Animals
}

\section{Celso Raul Romero Ramos, Mônica Magno Vilar*, Ana Lúcia Tabet Oller Nascimento, Paulo Lee Ho, Nilton Thaumaturgo*, Ricardo Edelenyi*, Marília Almeida**, Waldely de Oliveira Dias***, Catia Maria Diogo*, Miriam Tendler*/+}

\author{
Centro de Biotecnologia, Instituto Butantan, São Paulo, SP, Brasil *Departamento de Helmintologia, Instituto \\ Oswaldo Cruz-Fiocruz, Av. Brasil 4365, 21045-900 Rio de Janeiro, RJ, Brasil **MIP-CCB, Universidade \\ Federal de Santa Catarina, Florianópolis, SC, Brasil ***Instituto de Química, Universidade de São Paulo, \\ São Paulo, SP, Brasil
}

\begin{abstract}
Previous studies carried out with Sm14 in experimental vaccination against Schistosoma mansoni or Fasciola hepatica infections were performed with recombinant Sm14 (rSm14) produced in Escherichia coli by the pGEMEX system (Promega). The rSm14 was expressed as a $40 \mathrm{kDa}$ fusion protein with the major bacteriophage T7 capsid protein. Vaccination experiments with this $r S m 14$ in animal models resulted in consistent high protective activity against $\mathrm{S}$. mansoni cercariae challenge and enabled rSm14 to be included among the vaccine antigens endorsed by the World Health Organization for phase I/II clinical trials. Since the preparation of pGEMEX based rSm14 is time consuming and results in low yield for large scale production, we have tested other E. coli expression systems which would be more suitable for scale up and downstream processing. We expressed two different 6XHis-tagged Sm14 fusion proteins in a T7 promoter based plasmids. The 6XHis-tag fusions allowed rapid purification of the recombinant proteins through a $\mathrm{Ni}^{+2}$-charged resin. The resulted recombinant 18 and $16 \mathrm{kDa}$ proteins were recognized by anti-Sm14 antibodies and also by antiserum against adult $\mathrm{S}$. mansoni soluble secreted/excreted proteins in Western-Blot. Both proteins were also protective against $\mathrm{S}$. mansoni cercariae infection to the same extent as the rSm 14 expressed by the pGEMEX system.
\end{abstract}

Key words: Schistosoma mansoni - fatty acid binding protein - anti-helminth vaccine

Among parasitic infections, schistosomiasis is one of the most important diseases. This disease is a major cause of morbidity in the world. It ranks with malaria and tuberculosis in this regard. Up to 300 million people are infected with schistosomes. The infection is endemic to 76 countries where over 600 million people are at risk. Around $10 \%$ of the infected individuals present severe disease. In previous studies on the search for protective antigens against the Schistosoma mansoni cercariae infection, the characterization of Sm14 cDNA, which codes for a $14 \mathrm{kDa}$ protein, that belongs to the family of fatty acid-binding proteins (Moser et al. 1991), was described. The Sm14 cDNA was further ex-

\footnotetext{
This work was supported by Fiocruz, Fapesp, CNPq and Fundação Butantan.

${ }^{+}$Corresponding author. Fax: +55-21-2260-4866. E-mail: mtendler@ioc.fiocruz.br

Received 14 May 2001

Accepted 25 July 2001
}

pressed as a $40 \mathrm{kDa}$ fusion protein with the first 260 amino acids of the major T7 bacteriophage capsid protein using the pGEMEX vector system (Promega). The recombinant $40 \mathrm{kDa}$ protein was expressed in Escherichia coli, further fractionated and electroeluted from SDS-PAGE. This recombinant $\mathrm{Sm} 14$ preparation was shown to be protective in vaccinated animals upon challenge with $S$. mansoni cercariae and Fasciola hepatica metacercariae, which enables Sm14 to be included among the potential antigens for $S$. mansoni vaccine endorsed by WHO for fase I/II clinical tests (Tendler et al. 1996, Bergquist \& Colley 1998). However, the production of recombinant Sm14 using this protocol would restrict future assays aiming to develop an anti-helminth vaccine due to protein low yield and difficulties to scale-up this process. In order to develop a more suitable expression and purification system that would allow scale-up of the recombinant Sm14, we have changed the E. coli expression system. Here, we describe the results showing that the recombinant Sm14 produced by the new expression system was as protective as the Sm14 expressed by the pGEMEX system against $S$. mansoni cercariae challenge. 


\section{MATERIALS AND METHODS}

The pRSET A,B,C expression system was purchased from Invitrogen. The pET3-His (Chen \& Tsonwin 1994) was a generous gift from Dr Seiichi Yasuda from the National Institute of Genetic, Japan. All the reagents used here were of analytical grade.

Expression and purification of recombinant Sm14 - The recombinant Sm14 derived from $p$ GEMEX expression system (Promega) was purified as described (Tendler et al. 1996). The recombinant Sm14 proteins expressed by the pRSETA-Sm14, pET3-HisSm14 and pRSETA-6XHis-Sm14 constructs were obtained after transformation of chemically competent $E$. coli BL21(DE3). The transformed clones were grown in liquid LB (Luria Bertani medium) at $37^{\circ} \mathrm{C}$ with agitation $(200 \mathrm{rpm})$ until a 0.6 optical density was reached at $600 \mathrm{~nm}$. At this point, IPTG was added to a final concentration of $0.5 \mathrm{mM}$. The cultures were grown for an additional $3 \mathrm{~h}$ in the same conditions described and the cells were harvested by centrifugation at 2,000 g. The Sm14 was expressed in inclusion bodies in all the cases. The cells resuspended in $50 \mathrm{mM}$ Tris- $\mathrm{HCl} \mathrm{pH} 8.0,100 \mathrm{mM} \mathrm{NaCl}, 10 \mathrm{mM}$ EDTA, 10 mM 2-mercaptoethanol were disrupted by french pressure and the insoluble Sm14 was recovered by centrifugation. The inclusion bodies were washed by centrifugation with the previous solution also containing $2 \mathrm{M}$ urea and finally dissolved in $8 \mathrm{M}$ urea at room temperature for $2 \mathrm{~h}$ in the same buffer. The clarified supernantants were diluted 200 times by dropping in refolding solution $(50 \mathrm{mM}$ Tris$\mathrm{HCl} \mathrm{pH8.0,500} \mathrm{ml} \mathrm{NaCl}, 5 \mathrm{mM}$ imidazol) by stirring at room temperature for $18-24 \mathrm{~h}$. The total volume was clarified by centrifugation and loaded onto a C10 column (Amersham Pharmacia) containing $5 \mathrm{ml}$ of $\mathrm{Ni}^{+2}$-charged resin (Amersham Pharmacia) previously equilibrated with the refolding buffer at $1 \mathrm{ml} /$ min. The column was washed with 10-20 volumes of refolding buffer containing $20 \mathrm{mM}$ imidazol and the adsorbed protein was eluted by $1 \mathrm{M}$ imidazol in the refolding buffer. Fractions of $1 \mathrm{ml}$ were collected. Characterization of the fractions was done by SDSPAGE and Western-Blot according to described protocols (Harlow \& Lane 1988, Ausubel et al. 1989, Sambrook et al. 1989).

Immunization and challenge with S. mansoni cercariae - Groups of 10 female Swiss mice were immunized with the antigens in the presence of MPL-TDM Ribi adjuvant (Sigma). The animals were immunized in the foot pad on days 1, 7 and 14 and challenged subcutaneously with $100 \mathrm{~S}$. mansoni cercariae after 60 days of the third dose. The animals were further sacrified and the worms collected and counted after 45 days of the challenge as described (Tendler et al. 1996).

\section{RESULTS AND DISCUSSION}

In order to facilitate the expression, purification and scale up of recombinant $\mathrm{Sm} 14$, we digested the pGEMEX-Sm 14 construct with BamHI/HindIII, isolated and subcloned the Sm14 cDNA in the pRSET A vector (Invitrogen) in the same restriction sites. A second construct was done by isolating and subcloning the Sm14 cDNA fragment after XhoI/ HindIII digestion in the pET3-His (Chen \& Tsonwin 1994) in these same restriction sites. The resulting constructs were named pRSETA-Sm14 and pET3His-Sm14, respectivelly. The two plasmids allow the expression of the recombinant protein with a $6 \mathrm{XHis}$ tag fusion at N-terminus. However, in the case of the pRSET A-Sm14, the putative protein has a larger fusion peptide (37 amino acids versus 12 amino acids in the case of pET3-His-Sm14) with an enterokinase cleavage site between the recombinant protein and the fusion peptide (Fig. 1). A third construct was done by subcloning the NdeI/HindIII insert of pET3-His-Sm14 in pRSET A vector, resulting in the plasmid pRSET-His-Sm14. This third construct would express the same protein as pET3His-Sm14. However, since the plasmid copy number in the pRSET serie vectors is higher than $\mathrm{pET}$ vectors (results not shown), this feature allows higher plasmid yields from a single miniprep of pRSET-His-Sm14 than pET3-His-Sm14 vector, avoiding further steps of plasmid concentration for DNA sequencing reaction. Moreover, the higher copy number of pRSET-His-Sm14 plasmid would also yield more recombinant Sm14 than pET3-HisSm14 transformed cells.

Expression of recombinant Sm14 was achieved in these constructs by transformation and induction of E. coli BL21(DE3) by 0.5 M IPTG (Fig. 2). All the constructs were able to express the Sm14 protein. Due to the smaller peptide fusion (Figs 1, 2), a $16 \mathrm{kDa}$ band can be identified in the extracts of cells transformed with the pET3-His-Sm14 or pRSET-His-Sm14. In the case of the cells transformed with pRSET A-Sm14, a $18 \mathrm{kDa}$ band can be detected. In contrast, the cells transformed with the pGEMEX-Sm14 expressed a $40 \mathrm{kDa}$ protein, as a result of $\mathrm{Sm} 14$ fusion with the protein 10 of the bacteriophage T7 (Tendler et al. 1996). All these proteins were expressed as insoluble inclusion bodies (results not shown).

Using $\mathrm{Ni}^{+2}$-charged resin, it was possible to purify the two forms of recombinant Sm14 expressed by the three constructs (Fig. 1) as shown in Fig. 3, according to the protocol described in Materials and Methods. Western-blot analysis showed that both proteins reacted with anti-Sm14 antibodies raised against purified recombinant $\mathrm{Sm} 14$ expressed by the pGEMEX-Sm14 transformed E. coli (Fig. 2). 
Since this protein is composed by protein 10 of phage T7 fused to Sm14, we also tested if antibodies anti-protein 10 of phage T7 could react with recombinant Sm14 expressed by pRSET A-Sm14, pET3-His-Sm14 or pRSET-His-Sm14. As expected, the results were negative.
In the next step, we examined if animals immunized with these recombinant $\mathrm{Sm} 14$ could still provide protection against $S$. mansoni cercariae challenge. Female mice were immunized three times with 10 or 30 ug of Sm14 expressed by pRSET A-Sm14 or pRSET-His-Sm14 with or without MPL-TDM Ribi,

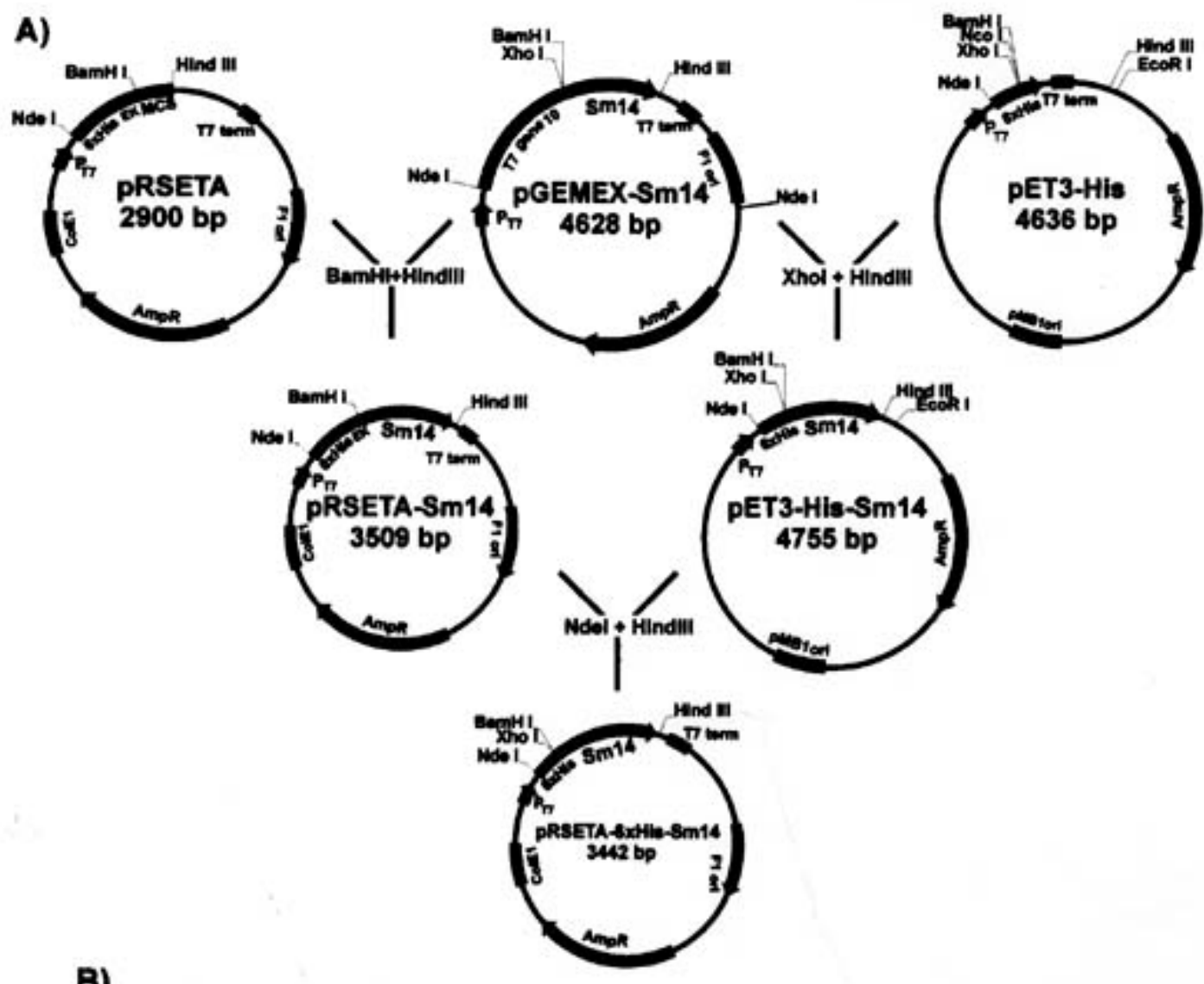

B)

\section{PRSETA-Sm14}

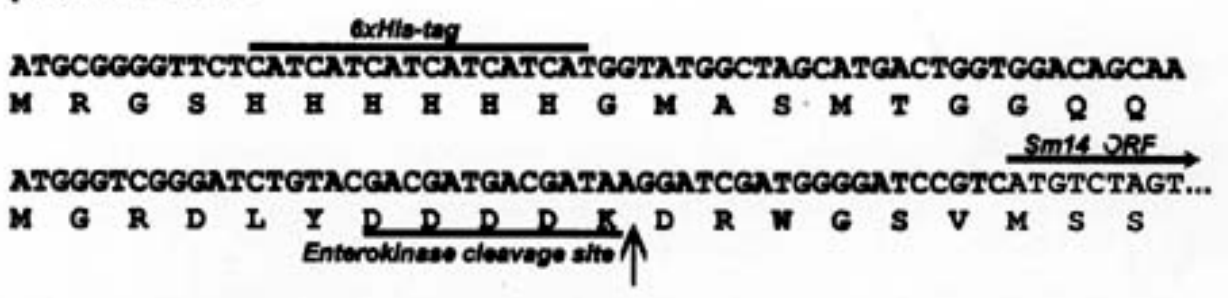

pET3-His-Sm14, pRSETA-6xHis-Sm14

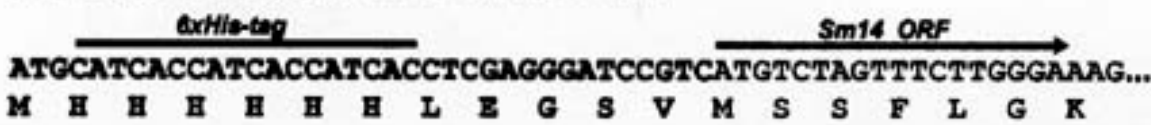

Fig. 1: schematic diagram showing the Sm14 cDNA subcloning in the different Escherichia coli expression vectors (A) and N-terminal amino acid sequences of the putative recombinant Sm14 expressed by the plasmids pRSETA-Sm14, pET3His-Sm14 and pRSET-His-Sm14 (B). 


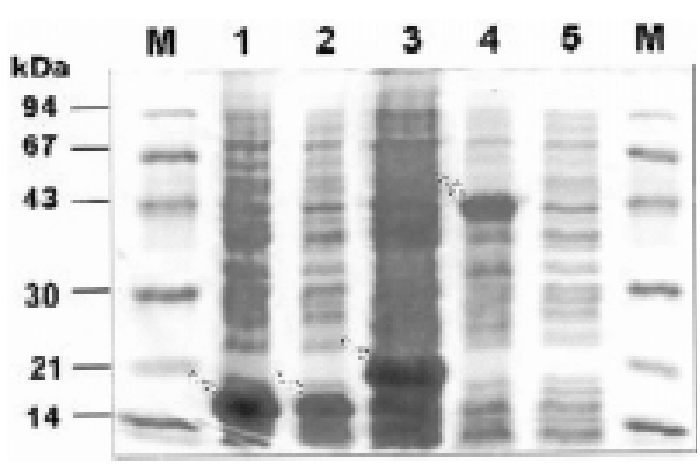

Fig. 2: polyacrylamide gel electrophoresis containing sodium dodecyl sulfate (SDS-PAGE) of bacterial extracts expressing recombinant Sm14. Extracts of Escherichia coli BL21(DE3) after induction with $0.5 \mathrm{mM}$ IPTG were fractionated in a $12 \%$ acrylamide/bisacrylamide (29:1) and stained with $0.1 \%$ Coomassie blue. The cells were transformed with the pRSET-His-Sm14 (lane 1), pET3-HisSm14, (lane 2), pRSETA-Sm14 (lane 3), pGEMEX-Sm14 (lane 4). Control cells were transformed with pRSETA plasmid (lane 5). M, protein standards. The recombinant Sm14 proteins are indicated by arrows.

as adjuvant, in a week interval between each immunization. Proteins secreted or excreted (SE) by adult worms incubated in phosphate buffered saline (PBS) were used as positive vaccine controls (Tendler et al. 1986). Two sets of experiments were done (Table). The new recombinant Sm14 antigens expressed by pRSET A-Sm14 and pRSET-His-Sm14 were equally effective in inducing protection in vaccinated mice to the same extent as that described for recombinant Sm14 expressed by pGEMEX-Sm14 (Tendler et al. 1996). This result also shows that the size and nature of the fusion peptide in the recombinant Sm14 were not important to elicite the protective response. They may be as large as the product of gene 10 of $\mathrm{T} 7$ bacteriophage or as small as a 12
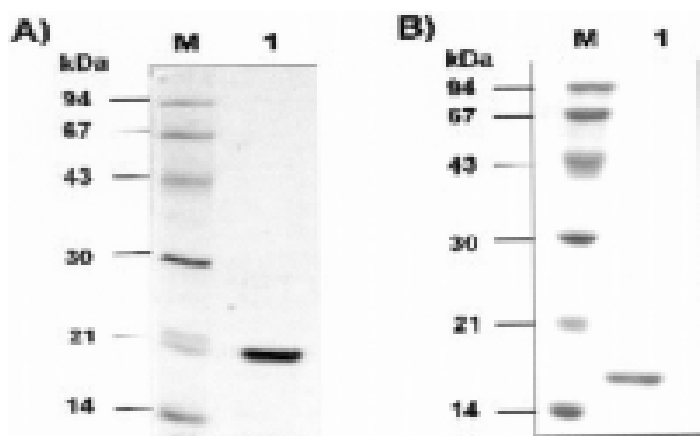

Fig. 3: polyacrylamide gel electrophoresis containing sodium dodecyl sulfate (SDS-PAGE) of purified recombinant Sm14 by $\mathrm{Ni}^{+}$-charged resin. The fractions containing the recombinant $\mathrm{Sm} 14$ were pooled, fractionated onto a $15 \%$ SDS-PAGE and stained with $0.1 \%$ Coomassie Blue. A pRSET A-Sm14 derived recombinant Sm14; B: pRSETHis-Sm14 derived recombinant Sm14. M: protein standards

amino acid peptide containing the 6XHis tag. The Sm14 based vaccine inhibited worm burden by 40 $55 \%$ when compared to non-vaccinated animals. This level of protection is comparable to other vaccine antigen candidates (Bergquist \& Colley 1998). The protective response elicited by Sm14 does not seem to be enhanced by MPL-TDM Ribi adjuvant. In experiment 2 , the lower protective value of SE might be due to the low number of animals used. Based on these results, Sm14-derived from these new constructs confirmed the previous evidence that Sm14 is a potential antigen capable of stimulating protective immunity against S. mansoni and $F$. hepatica (not shown here) infection and was approved for further phase I/II trials for vaccine development against both schistosomiasis and fascioliasis.

TABLE

Protective activity of recombinant Sm14 against Schistosoma mansoni cercariae challenge in Swiss mice

\begin{tabular}{|c|c|c|c|c|}
\hline & $\begin{array}{l}\text { Antigen } \\
(3 \text { doses })\end{array}$ & $\begin{array}{c}\text { Number of } \\
\text { animals }\end{array}$ & $\begin{array}{l}\text { Worm } \\
\text { burden }\end{array}$ & $\begin{array}{l}\text { Protection } \\
(\%)\end{array}$ \\
\hline Exp. 1 & $\begin{array}{l}10 \mu \mathrm{g} \mathrm{Sm} 14^{\mathrm{A}} \\
10 \mu \mathrm{g} \mathrm{Sm} 14^{\mathrm{A}}+\text { Ribi } \\
\text { SE } \\
\text { Ribi } \\
\text { PBS }\end{array}$ & $\begin{array}{c}10 \\
10 \\
12 \\
8 \\
12\end{array}$ & $\begin{array}{c}12 \\
10.2 \\
12.4 \\
19.9 \\
21.4\end{array}$ & $\begin{array}{c}43.9 \\
52.3 \\
42 \\
7 \\
0\end{array}$ \\
\hline Exp. 2 & $\begin{array}{l}10 \mu \mathrm{g} \mathrm{Sm} 14^{\mathrm{H}}+\text { Ribi } \\
30 \mu \mathrm{g} \mathrm{Sm} 14^{\mathrm{H}}+\text { Ribi } \\
\text { SE } \\
\text { Ribi } \\
\text { PBS }\end{array}$ & $\begin{array}{l}10 \\
9 \\
7 \\
7 \\
7\end{array}$ & $\begin{array}{c}11.9 \\
12.7 \\
18 \\
22.3 \\
26.3\end{array}$ & $\begin{array}{c}54.8 \\
51.7 \\
26.6 \\
15.2 \\
0\end{array}$ \\
\hline
\end{tabular}

$\overline{\mathrm{A}, \mathrm{H}} \mathrm{Sm} 14$ derived from Escherichia coli cells transformed with pRSET A-Sm14 or pRSET-His-Sm14 plasmid, respectively. 


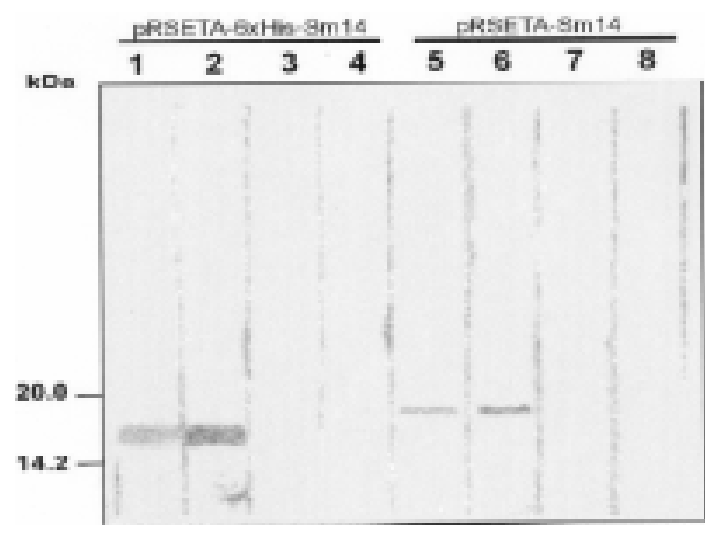

Fig. 4: Western-Blot of recombinant $\mathrm{Sm} 14$ with antibodies anti-Sm14. Purified recombinant Sm14 antigens expressed by pRSET-His-Sm14 (lanes 5, 6, 7, 8) or pRSETA-Sm14 (lanes 1, 2, 3, 4) were fractionated onto a 15\% SDS-PAGE and electrotransferred to a nitrocellulose sheet. After staining with $0.1 \%$ Ponceau S solution, the the lanes were cut and incubated with antibodies anti-Sm14 raised in rabbits against pGEMEX-Sm14 derived recombinant Sm14 (lanes 1 and 5), with antibodies against SE (lanes 2 and 6), with antibodies against protein 10 of bacteriophage T7 (lanes 3 and 7) and with pre-immune rabbit antiserum (lane 4 and $8)$.

\section{REFERENCES}

Ausubel FM, Brent R, Kingston RE, Moore DD, Seidman JG, Smith JA, Sthrul K 1989. Current Protocols in Molecular Biology, John Wiley \& Sons, New York.

Bergquist NR, Colley DG 1998. Schistosomiasis vaccines: research to development. Parasitol Today 14: 99-104.

Chen BP, Tsonwin H 1994. Expression vector for affinity purification and radiolabeling of proteins using Escherichia coli as host. Gene 139: 73-75.

Harlow E, Lane D 1988. Antibodies: A Laboratory Manual, Cold Spring Harbor Laboratory Press, Cold Spring Harbor.

Moser D, Tendler M, Griffiths G, Klinkert MQ 1991. A 14-kDa Schistosoma mansoni polypeptide is homologous to a gene family of fatty acid binding proteins. J Biol Chem 266: 8447-8454.

Sambrook J, Fritsch EF, Maniatis T 1989. Molecular Cloning: A Laboratory Manual, 2nd ed. Cold Spring Harbor Laboratoy, Cold Spring Harbor.

Tendler M, Brito CA, Vilar MM, Serra-Freire N, Diogo CM, Almeida MS, Delbem ACB, Silva JF, Savino W, Garratt RC, Katz N, Simpson AJG 1996. A Schistosoma mansoni fatty acid-binding protein, Sm14, is the potential basis of a dual-purpose anti-helminth vaccine. Proc Natl Acad Sci USA 93: 269-273.

Tendler M, Pinto RM, Lima AO, Gebara G, Katz N 1986. Schistosoma mansoni: vaccination with adult worm antigens. Int J Parasitol 16: 347-352. 
136 r-Sm14 - pRSETA Efficacy in Experimental Animals - Celso Raul Romero Ramos et al. 